\title{
Glycaemic and insulin responses, glycaemic index and insulinaemic index values of rice between three Asian ethnic groups
}

\author{
V. M. H. Tan ${ }^{1,2 *}$, T. Wu ${ }^{1}$, C. J. Henry ${ }^{1,3}$ and Y. S. Lee ${ }^{1,2,4}$ \\ ${ }^{1}$ Clinical Nutrition Research Centre, Singapore Institute for Clinical Sciences, A*STAR, Singapore \\ ${ }^{2}$ Department of Paediatrics, Yong Loo Lin School of Medicine, National University of Singapore, Singapore \\ ${ }^{3}$ Department of Biochemistry, Yong Loo Lin School of Medicine, National University of Singapore, Singapore \\ ${ }^{4}$ Division of Endocrinology and Diabetes, Khoo Teck Puat-National University Children's Medical Institute, \\ National University Hospital, National University Health System, Singapore
}

(Submitted 13 November 2014 - Final revision received 12 January 2015 - Accepted 4 February 2015 - First published online 19 March 2015)

\section{Abstract}

Asians exhibit larger glycaemic response (GR) and insulin response (IR) than Caucasians, predisposing to an increased risk of type 2 diabetes mellitus (T2DM). We aimed to determine the GR and IR as well as the glycaemic index (GI) and insulinaemic index (II) of two rice varieties among three ethnic groups in Singapore. A total of seventy-five healthy males (twenty-five Chinese, twenty-five Malay and twenty-five Asian-Indians) were served the available equivalent carbohydrate amounts ( $50 \mathrm{~g}$ ) of test foods (Jasmine rice and Basmati rice) and a reference food (glucose) on separate occasions. Postprandial blood glucose and plasma insulin concentrations were measured at fasting ( -5 and $0 \mathrm{~min}$ ) and at 15, 30, 45, 60, 90 and $120 \mathrm{~min}$ after food consumption. Using the trapezoidal rule, GR, IR, GI and II values were determined. The GR did not differ between ethnic groups for Jasmine rice and Basmati rice. The IR was consistently higher for Jasmine rice $(P=0.002)$ and Basmati rice $(P=0.002)$ among Asian-Indians, probably due to compensatory hyperinsulinaemia to maintain normoglycaemia. The GI and II of both rice varieties did not differ significantly between ethnicities. The overall mean GI for Jasmine rice and Basmati rice were 91 (SD 21) and 59 (SD 15), respectively. The overall mean II for Jasmine rice was 76 ( $\mathrm{SD}$ 26) and for Basmati rice was 57 (SD 24). We conclude that the GI values presented for Jasmine rice and Basmati rice were applicable to all three ethnic groups in Singapore. Future studies should include deriving the II for greater clinical utility in the prevention and management of $\mathrm{T} 2 \mathrm{DM}$.

Key words: Glycaemic response: Insulin response: Glycaemic index: Jasmine rice: Basmati rice: Ethnic groups: Asians

Numerous studies have shown that Asians, Chinese and nonCaucasians exhibit a 2-3-fold larger postprandial glycaemic response (GR) and insulin response (IR) than Caucasians ${ }^{(1-4)}$. In a recent study, GR following rice consumption is over $60 \%$ greater among Chinese compared with Europeans ${ }^{(5)}$. Higher blood glucose concentrations stimulate increased production of insulin, resulting in a state of hyperinsulinaemia. The chronically increased insulin demand may eventually result in pancreatic $\beta$-cell failure and, as a consequence, impaired glucose tolerance, leading to type 2 diabetes mellitus $(\mathrm{T} 2 \mathrm{DM})^{(6)}$.

Rice is a staple carbohydrate of many Asian populations, providing up to two-thirds of daily energy intake in Southeast Asian countries ${ }^{(5)}$, making it a major contributor to the overall glycaemic load of most Asian diets ${ }^{(7-10)}$. The extent to which different varieties of rice influence postprandial glycaemia has potential relevance in the prevention and treatment of obesity and T2DM in Asia, where the recent increase in prevalence outweighs other parts of the world ${ }^{(11)}$. In many studies, rice consumption, especially white rice, has been implicated in the deterioration of glucose metabolism, which leads to an increased risk of $\mathrm{T}_{2} \mathrm{DM}^{(9,10,12)}$. This is probably mediated by the glycaemic potency of rice, as measured by their dietary glycaemic index (GI), a classification of carbohydrates according to their effect on postprandial glycaemia $^{(13,14)}$.

The question whether GI varies between ethnicities is equivocal. Some studies have shown no ethnic differences in GI values ${ }^{(3,15)}$; however, other studies have demonstrated significant effects of ethnicity on the GI value of white bread $^{(16)}$ and rice ${ }^{(5)}$. One of the major impediments in the use of GI tables worldwide has been the uncertainty of the applicability to different ethnic groups. If the standard

Abbreviations: GI, glycaemic index; GR, glycaemic response; HOMA-B, homeostatic model assessment- $\beta$ cell function; HOMA-IR, homeostatic model assessment-insulin resistance; IAUC, incremental AUC; II, insulinaemic index; IR, insulin response; T2DM, type 2 diabetes mellitus. 
classifications of low, medium and high GI differ between Asians of different ethnicities, this has important implications for dietary recommendations for individuals living in a multiethnic Asian population, especially for those with T2DM. Insulinaemic index (II), derived similar to GI, measures the extent to which a food raises plasma insulin levels ${ }^{(13,17,18)}$. The insulinaemic effects of foods may be relevant to the prevention of T2DM as prolonged postprandial insulinaemia may play a role in the pathogenesis of $\mathrm{T}_{2} \mathrm{DM}^{(19)}$ and associated chronic diseases ${ }^{(20,21)}$.

The population in Singapore comprises three major ethnic groups: Chinese; Malays; Asian-Indians. Yet, there are significant differences in the prevalence of obesity and T2DM between our three ethnic groups. The Malay population has the highest prevalence of obesity $(24.0 \%)$, while our Asian-Indian population has the highest prevalence of T2DM $(17 \cdot 2 \%)^{(22)}$. This offers us an unusual opportunity to study the effect of different Asian ethnicities on postprandial GR and IR as well as GI and II values to the same food.

The aims of the present study were to examine GR and IR as well as to determine the GI and II of two varieties of commonly consumed rice among the ethnic groups in Singapore. This is the first study known to the authors to examine the role of three ethnic groups residing in a shared and common multiethnic Asian environment. The results obtained will provide insights into the metabolic effects of consuming the same food among the different ethnic groups.

\section{Methods}

\section{Subjects}

A total of seventy-five healthy male participants, consisting of twenty-five Chinese, twenty-five Malays and twenty-five Asian-Indians, were recruited for the present study. The participants initially underwent a screening visit to assess eligibility, which comprised of a health assessment, where anthropometric measurements (Table 1) were taken and a health questionnaire (relating to medical history, smoking habits, history of any illness and use of any medications) was administered. All anthropometric measurements were made at least $2 \mathrm{~h}$ after food consumption. Height and weight were measured without shoes by using a digital scale cum stadiometer (SECA 763). A digital blood pressure monitor (Omron HEM-907) was used to obtain blood pressure readings. Waist circumference was measured using a standard measuring tape (SECA tape measure) at the midpoint between the coastal margins of the ribs and the upper margin of iliac crest and the hip circumference at the widest level of the greater trochanters on both sides. Percentage of body fat was measured by air displacement plethysmography method (Bod Pod; Cosmed).

Those who fulfilled all acceptable criteria (BMI 18.5 to $<25 \mathrm{~kg} / \mathrm{m}^{2}$, age $18-45$ years, both parents and grandparents were of the same ethnicity, blood pressure 110-120/ $70-90 \mathrm{mmHg}$, fasting blood glucose $4-6 \mathrm{mmol} / \mathrm{l}$, not on prescription medication, non-smoking, and no genetic or metabolic diseases) were included in the present study. On the day before the test visit, participants were asked to avoid intake of alcohol, restrict caffeine-containing drinks, refrain from intense strenuous physical activity and to consume their usual meals. Participants were also requested to fast overnight for $10-12 \mathrm{~h}$ before each test visit.

The present study was conducted according to the guidelines laid down in the Declaration of Helsinki, and all procedures involving human subjects were approved by the National Healthcare Group Domain Specific Review Board, Singapore. All the participants provided written informed consent before their participation in the study. This was registered at clinicaltrials.gov as NCT01804738.

\section{Study procedures}

A randomised, cross-over, within-subject, repeated-measure non-blind design was adopted. Participants arrived at the research centre between 08.00 and 09.00 hours after $10-12 \mathrm{~h}$

Table 1. Anthropometric characteristics by ethnicity

(Mean values and standard deviations)

\begin{tabular}{|c|c|c|c|c|c|c|c|}
\hline & \multicolumn{2}{|c|}{ Chinese ( $n$ 25) } & \multicolumn{2}{|c|}{ Malay $(n 25)$} & \multicolumn{2}{|c|}{ Asian-Indians ( $n$ 25) } & \multirow[b]{2}{*}{$P^{\star}$} \\
\hline & Mean & SD & Mean & SD & Mean & SD & \\
\hline Age (years) & $23 \cdot 0$ & $1 \cdot 3$ & $23 \cdot 0$ & $2 \cdot 3$ & $24 \cdot 1$ & $2 \cdot 0$ & 0.072 \\
\hline Weight (kg) & $63 \cdot 8$ & $6 \cdot 9$ & $65 \cdot 8$ & $7 \cdot 1$ & $68 \cdot 7$ & 8.5 & 0.073 \\
\hline Height $(\mathrm{cm})$ & $172 \cdot 6$ & $6 \cdot 0$ & $170 \cdot 8^{\mathrm{a}}$ & $6 \cdot 0$ & $176 \cdot 2^{b}$ & $7 \cdot 0$ & 0.012 \\
\hline BMI $\left(\mathrm{kg} / \mathrm{m}^{2}\right)$ & 21.4 & 1.8 & 22.5 & 1.7 & $22 \cdot 1$ & 1.9 & 0.085 \\
\hline Systolic blood pressure $(\mathrm{mmHg})$ & 125 & 11 & 127 & 8 & 124 & 7 & 0.528 \\
\hline Diastolic blood pressure $(\mathrm{mmHg})$ & 76 & 9 & 73 & 8 & 72 & 10 & 0.247 \\
\hline Mean fasting glucose $(\mathrm{mmol} / \mathrm{l})$ & 4.55 & 0.31 & 4.46 & 0.25 & 4.54 & 0.32 & 0.484 \\
\hline Mean fasting insulin (mU/l†) & 7.43 & $2 \cdot 85$ & $9 \cdot 10$ & 3.38 & $8 \cdot 10$ & 3.93 & 0.225 \\
\hline Waist circumference (cm) & $75 \cdot 5$ & 5.4 & $72 \cdot 7^{\mathrm{a}}$ & 4.4 & $77 \cdot 7^{\mathrm{b}}$ & 6.9 & 0.010 \\
\hline Body fat (\%) & $16 \cdot 0^{\mathrm{a}}$ & 5.9 & $17 \cdot 8$ & $5 \cdot 2$ & $20 \cdot 5^{\mathrm{b}}$ & $6 \cdot 8$ & 0.035 \\
\hline Mean HOMA-IR & $1.45^{\mathrm{a}}$ & 0.53 & $1.43^{\mathrm{a}}$ & 0.51 & $2 \cdot 16^{\mathrm{b}}$ & 0.89 & $<0.001$ \\
\hline Mean HOMA-B & $165 \cdot 7^{\mathrm{a}}$ & $86 \cdot 1$ & $172 \cdot 6$ & $76 \cdot 8$ & $229 \cdot 2^{\mathrm{b}}$ & $93 \cdot 3$ & 0.020 \\
\hline
\end{tabular}

HOMA-IR, homeostatic model assessment-insulin resistance; HOMA-B, homeostatic model assessment- $\beta$ cell function.

a,b Mean values within each row with unlike superscript letters were significantly different $(P<0 \cdot 05)$.

${ }^{*} P$ values represent comparison between the ethnic groups.

† To convert insulin from $\mathrm{mU} / \mathrm{l}$ to $\mathrm{pmol} / \mathrm{l}$, multiply by 6.945 . 
of overnight fast. Following a $10 \mathrm{~min}$ rest, two fasting blood samples were obtained $5 \mathrm{~min}$ apart for determining baseline blood glucose and insulin levels. After that, participants were given either a reference food or a test food with water to consume at a comfortable pace within $15 \mathrm{~min}$. The reference food consisted of $50 \mathrm{~g}$ of glucose anhydrous powder dissolved in $250 \mathrm{ml}$ water, while the test food was Jasmine rice (Double FP Thai Hom Mali premium quality fragrant rice, Thailand) or Basmati rice (Dreamrice ${ }^{\mathrm{TM}}$, Singapore). These two rice varieties were chosen as they were commonly consumed by the local population. Jasmine rice was tested locally to be classified as high $\mathrm{GI}^{(23)}$, while Basmati rice was tested in a local laboratory and certified as low GI on its product packaging. Both rice samples were served in portions containing $50 \mathrm{~g}$ of available carbohydrates (difference between total carbohydrates and dietary fibre). This amounted to $63.6 \mathrm{~g}$ (with $130 \mathrm{ml}$ water) and $66.5 \mathrm{~g}$ (with $170 \mathrm{ml}$ water) of uncooked Jasmine rice and Basmati rice, respectively, cooked in individual portions in a rice cooker (Iona GL12 rice cooker, Singapore) and served with $250 \mathrm{ml}$ water. In total, the reference food was tested thrice, and the test food was tested once by each participant in a randomised order. This is in accordance with $\mathrm{FAO} / \mathrm{WHO}$ recommendations ${ }^{(24)}$ for the determination of GI value that each subject will test each test food once and the reference food thrice in a random order on separate days, with at least $2 \mathrm{~d}$ gap between measurements to minimise carry-over effects.

After the commencement of eating, further blood samples were taken at 15, 30, 45, 60, 90 and $120 \mathrm{~min}$. The protocol used to measure blood glucose response was adopted from that described by Brouns et al. ${ }^{(25)}$ and is in line with the $\mathrm{FAO} / \mathrm{WHO}$ recommendations ${ }^{(24)}$. Blood sample was obtained by finger prick using sterile, single-use lancing device (Accucheck Safe-T-Pro Plus; Roche Diagnostics). Before a finger prick, participants were encouraged to warm their hand to increase blood flow. To minimise plasma dilution, fingertips were not squeezed to extract blood but were gently massaged starting from the base of the hand moving towards the tips. The first two drops of expressed blood were discarded, and the next drop was used for testing. Blood glucose was measured using the HemoCue 201+ Glucose analyzer (HemoCue 201 RT).

For the measurement of blood insulin levels at each time point, after blood glucose measurement, $300 \mu \mathrm{l}$ of capillary blood (obtained from finger pricks) was collected into Microvette ${ }^{\circledR}$ capillary blood collection tubes treated with di-potassium EDTA (CB 300 K2E; Sarstedt) and stored in crushed ice immediately until the end of the study visit. The Microvette ${ }^{\circledR}$ tubes were centrifuged at $4500 \mathrm{rpm}$ for $12 \mathrm{~min}$ (Rotina 420R; Hettich), and the supernatant plasma was transferred into individually labelled $1.5 \mathrm{ml}$ microtubes (Axygen Scientific, Inc.), which were immediately transferred to a freezer held at $-80^{\circ} \mathrm{C}$ until analysis of insulin. Plasma insulin concentrations were determined by electrochemiluminescence immunoassay using an automated analyzer (Cobas E411; Roche Diagnostics). The Cobas system is a reliable method of blood insulin determination ${ }^{(26)}$.

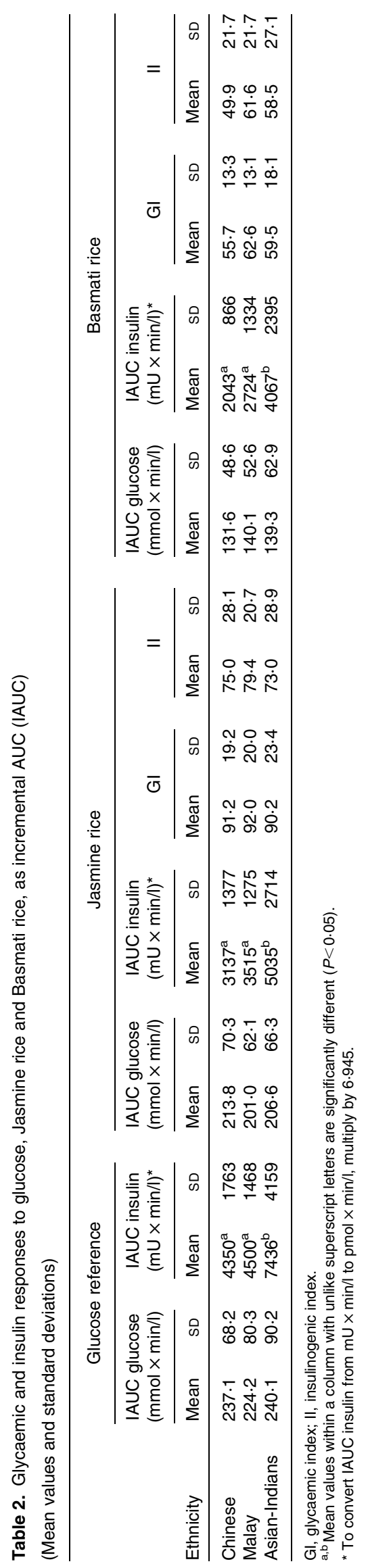




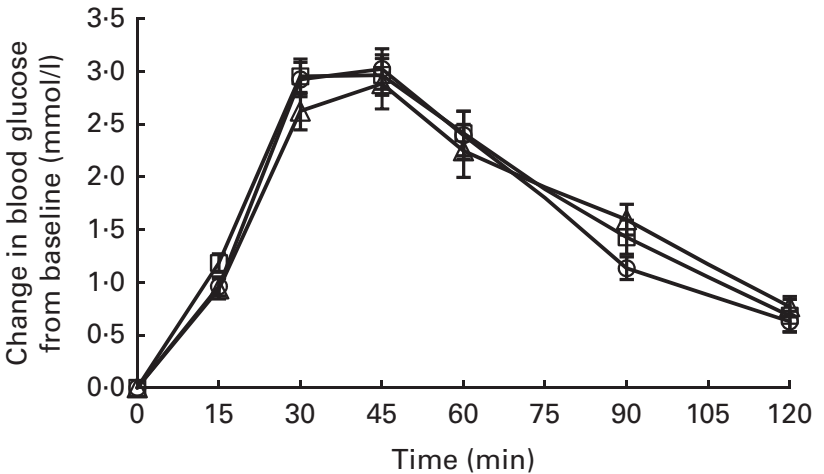

Fig. 1. Temporal blood glucose response curves for Jasmine rice among Chinese $(\square)$, Malays $(\bullet)$ and Asian-Indians $(\triangle)$. Data are represented as change in glucose response from baseline over $120 \mathrm{~min}$ following the consumption of Jasmine rice. Values are means, with their standard errors represented by vertical bars. Repeated-measures ANOVA with post hoc Bonferroni multiple-comparison tests revealed an overall difference between ethnic groups $(P=0.757)$.

We computed homeostatic model assessment-insulin resistance (HOMA-IR) as follows:

fasting insulin $(\mathrm{mU} / \mathrm{l}) \times$ fasting glucose $(\mathrm{mmol} / \mathrm{l}) / 22.5$.

Homeostatic model assessment- $\beta$ cell function (HOMA-B) was calculated using the following formula:

$(20 \times$ fasting insulin $(\mathrm{mU} / \mathrm{l})) /($ fasting glucose $(\mathrm{mmol} / \mathrm{l})-3.5)$. (To convert insulin in $\mathrm{mU} / 1$ to $\mathrm{pmol} / \mathrm{l}$, multiply by 6.945 ).

\section{Statistical analyses}

One study on ethnic differences in postprandial glycaemia ${ }^{(3)}$ between UK Caucasians and Asian Indians has observed at least a $59 \mathrm{mmol} / \mathrm{min}$ per litre difference in GR between the two groups with standard deviations of 79 and $47 \mathrm{mmol} / \mathrm{min}$ per litre, respectively. At the $5 \%$ level of significance with power of $80 \%$, a minimum sample size of twenty-one subjects for each group would be required. Studies on in vivo assessment of GI have been based on ten subjects, to take into account inter-individual variations ${ }^{(24,25)}$. As we were investigating ethnic differences, a sample size of twenty-five from each ethnic group (total sample size of seventy-five) was considered adequate for the present study.

The GR data was analysed using 'change in GR', which was calculated by taking the difference between the blood glucose reading at each time point and mean baseline blood glucose value (based on two baseline values taken at $5 \mathrm{~min}$ apart). This represented the relative increment in GR at any time point compared with the baseline value. The total GR over 120 min was expressed as the incremental AUC (IAUC) ignoring the area under the baseline using the trapezoidal rule $^{(24,25)}$. The IR data was analysed in the same manner as the GR data, using 'change in IR'. These data were then used to construct IR curves and calculate the IAUC (using the trapezoidal rule).

The intention-to-treat analysis included data from all the participants. All physiologically valid data were included in the computation of GI and II. In the calculation of GI, the absolute IAUC glucose values for each rice variety was expressed as a percentage of the mean IAUC glucose values of the reference food, and the resulting values were averaged to obtain the GI value for each rice variety. The II, which measures the IR of food, was calculated similar to GI, where the absolute IAUC insulin values for each rice variety were expressed as a percentage of the mean IAUC glucose values of the reference food.

Results were expressed as means with standard deviations where appropriate. Differences in GR and IR and GI values were analysed using one-way ANOVA with post hoc Bonferroni correction for multiple comparisons. The temporal GR and IR over $120 \mathrm{~min}$ were analysed using repeated-measures ANOVA with post hoc Bonferroni correction. Paired $t$ tests were used to compare the IAUC and rates of decline from 45 to $60 \mathrm{~min}$, 60 to $90 \mathrm{~min}$ and 90 to $120 \mathrm{~min}$ for Jasmine rice and Basmati rice. Correlation analyses were conducted to determine the relationships between GI, II with body composition (waist circumference and percentage of body fat) and metabolic indices (HOMA-IR and HOMA-B). Statistical analyses were conducted using the SPSS version 19 (SPSS, Inc.). A $P$ value $<0.05$ was considered statistically significant.

\section{Results}

Table 1 compares the characteristics of the study participants by ethnicity. There were no differences in age, BMI, blood pressure, fasting glucose level and fasting insulin level between the three groups. However, waist circumference $(P=0.010)$ and percentage of body fat $(P=0.035)$ were significantly higher among the Asian-Indians compared with Chinese and Malays. The HOMA-IR, a marker of insulin resistance, was highest for Asian-Indians $(P<0 \cdot 001)$ compared with Chinese and Malays. In parallel with HOMA-IR, Asian-Indians showed a significantly higher HOMA-B $(P=0 \cdot 020)$ compared with Chinese and Malays. This indicated a compensatory hyperinsulinaemia in the presence of greater insulin resistance among Asian-Indians to maintain fasting normoglycaemia.

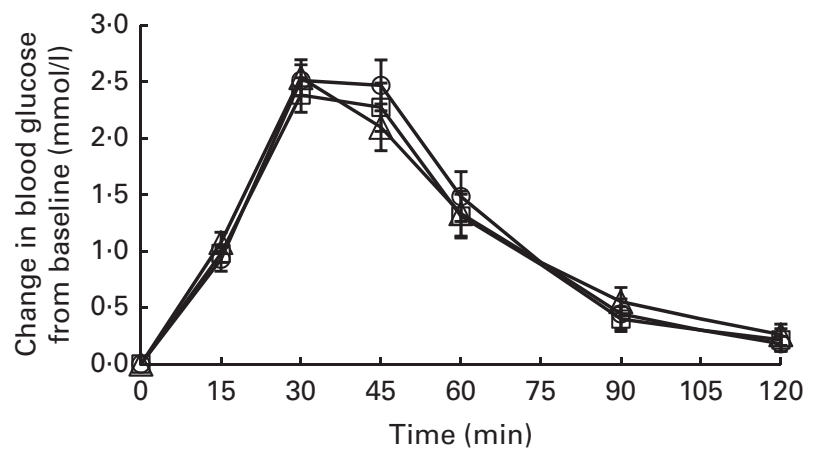

Fig. 2. Temporal blood glucose response curves for Basmati rice among Chinese $(\square)$, Malays $(\bullet)$ and Asian-Indians $(\triangle)$. Data are represented as change in glucose response from baseline over $120 \mathrm{~min}$ following the consumption of Basmati rice. Values are means, with their standard errors represented by vertical bars. Repeated-measures ANOVA with post hoc Bonferroni multiple-comparison tests revealed an overall difference between ethnic groups $(P=0.837)$. 


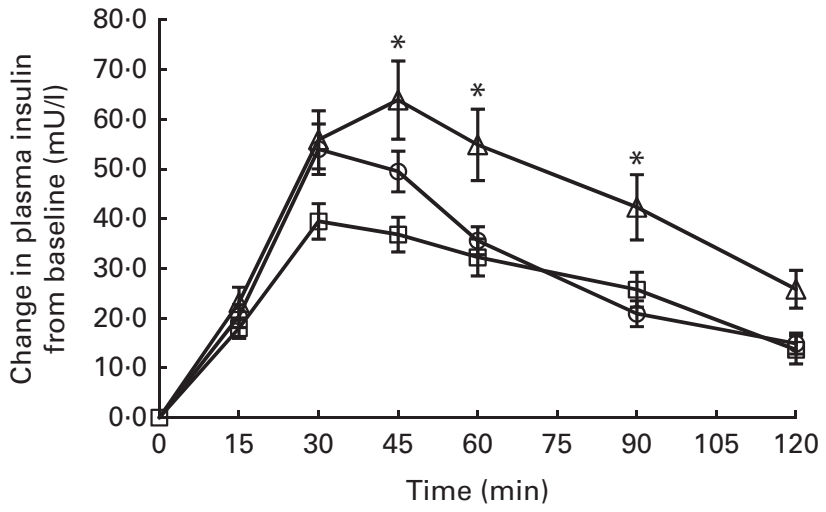

Fig. 3. Temporal plasma insulin response curves for Jasmine rice among Chinese $(\square)$, Malays $(-)$ and Asian-Indians $(\triangle)$. Data are represented as change in insulin response from baseline over $120 \mathrm{~min}$ following the consumption of Jasmine rice. Values are means, with their standard errors represented by vertical bars. * Mean value was significantly different at a specific time point $(P<0.05)$. Repeated-measures ANOVA with post hoc Bonferroni multiple-comparison tests revealed an overall difference between ethnic groups $(P=0.002)$. To convert insulin from $\mathrm{mU} / \mathrm{l}$ to $\mathrm{pmol} / \mathrm{l}$, multiply by 6.945 .

The GR IAUC did not differ between ethnic groups for glucose reference, Jasmine rice and Basmati rice (Table 2). The mean intra-individual variations in GR to the three glucose reference were $17 \cdot 3 \% \mathrm{CV}$ for Chinese, $17 \cdot 1 \% \mathrm{CV}$ for Malays and $16.0 \% \mathrm{CV}$ for Asian-Indians $(P=0.841)$. These values were consistent with previously reported variability for repeat tests of the reference foods in normal subjects ${ }^{(3,27,28)}$. The inter-individual variations in GR to the reference food were $28.8 \% \mathrm{CV}$ for Chinese, $35.8 \% \mathrm{CV}$ for Malays and $37.6 \% \mathrm{CV}$ for Asian-Indians. There were also no significant differences in temporal GR patterns for the three groups for Jasmine rice $\left(P_{\text {interaction }}=0.757\right.$; Fig. 1$)$ and Basmati rice $\left(P_{\text {interaction }}=0.837 ;\right.$ Fig. 2$)$. Basmati rice showed an initial faster rate of decline from 45 to $60 \mathrm{~min}$ (mean difference $2.2 \mathrm{mmol} / 1$ per $\mathrm{min} ; P=0.002$ ) than Jasmine rice. However, the later postprandial rate of decline was significantly more rapid for Jasmine rice from 90 to 120 min (mean difference $6.7 \mathrm{mmol} / 1$ per min; $P<0.001)$ compared with Basmati rice.

In contrast, IR between the ethnic groups showed significant differences. The insulin IAUC was consistently higher in Asian-Indians compared with Chinese and Malays for glucose reference $(P<0 \cdot 001)$, Jasmine rice $(P=0 \cdot 002)$ and Basmati rice $(P<0.001)$. The temporal IR patterns for Jasmine rice and Basmati rice differed considerably between the ethnic groups (Figs. 3 and 4). Time point analyses showed that the IR for Jasmine rice at 45, 60, 90 and 120 min (Fig. 3) were significantly higher for Asian-Indians than for Chinese and Malay. For basmati rice, the IR at 15, 30, 45, 60 and $90 \mathrm{~min}$ (Fig. 4) were also significantly higher for Asian-Indians compared with Chinese and Malays.

As shown in Table 2 , the GI of Jasmine rice $(P=0.957)$ and Basmati rice $(P=0.277)$ did not differ between the ethnic groups. The overall mean GI was 91.1 (SD 20.7) (high GI) for Jasmine rice and $59 \cdot 3$ (SD 15.1) (medium GI) for Basmati rice. The GI values for both rice varieties were not significantly different between the ethnic groups after adjusting for body fat or waist circumference. Similarly, the II for Jasmine rice $(P=0.681)$ and Basmati rice $(P=0.197)$ were not different between the ethnic groups. The overall mean II for Jasmine rice was $75 \cdot 8$ (SD 25.9) and Basmati rice was 56.7 (SD 23.8). Adjustment for body composition (body fat or waist circumference) made no difference to the II values between the ethnic groups.

Correlation analyses indicated that GI and II for Jasmine rice and Basmati rice were not related to any of the body composition measurements (waist circumference and percentage of body fat) or metabolic indices (HOMA-IR and HOMA-B) $(P>0 \cdot 05)$. These results demonstrate that GI and II were independent of individuals' body composition and metabolic status, making them valid properties of food.

Between rice varieties, we observed that Jasmine rice showed consistently higher GR and IR as well as significantly higher GI $(P=0 \cdot 001)$ and II $(P<0 \cdot 001)$ values (Table 2$)$ compared with Basmati rice for each ethnic group (Figs. 5 and 6).

\section{Discussion}

The present study was the first to examine the role of three ethnicities in a multi-ethnic Asian environment on postprandial GR and IR as well as GI and II values to two commonly consumed rice varieties. We observed no ethnic variations in GR to Jasmine rice and Basmati rice; yet, there were considerable differences in IR.

Even though there were no differences in fasting glucose and fasting insulin levels, Asian-Indians mounted significantly higher insulin excursions for both Jasmine rice and Basmati rice compared with Chinese and Malays, even after adjustment for percentage of body fat or waist circumference. These differences in IR were probably due to a compensatory hyperinsulinaemia in the presence of greater insulin resistance among the Asian-Indians to maintain fasting normoglycaemia. Genetically, Asian-Indians have a high prevalence of insulin

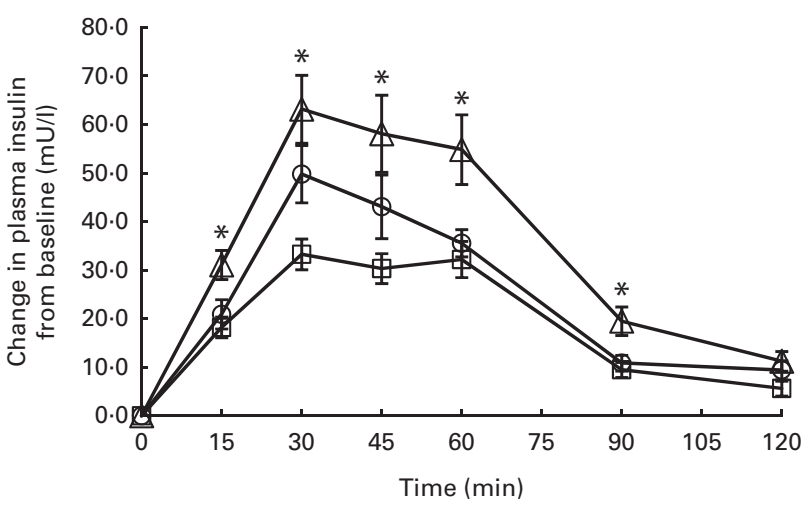

Fig. 4. Temporal plasma insulin response curves for Basmati rice among Chinese $(\square)$, Malays ( $\bullet)$ and Asian-Indians $(\triangle)$. Data are represented as change in insulin response from baseline over $120 \mathrm{~min}$ following the consumption of Basmati rice. Values are means, with their standard errors represented by vertical bars. ${ }^{*}$ Mean value was significantly different at a specific time point $(P<0.05)$. Repeated-measures ANOVA with post hoc Bonferroni multiple-comparison tests revealed an overall difference between ethnic groups $(P<0.001)$. To convert insulin from $\mathrm{mU} / \mathrm{l}$ to $\mathrm{pmol} / \mathrm{l}$, multiply by 6.945 . 


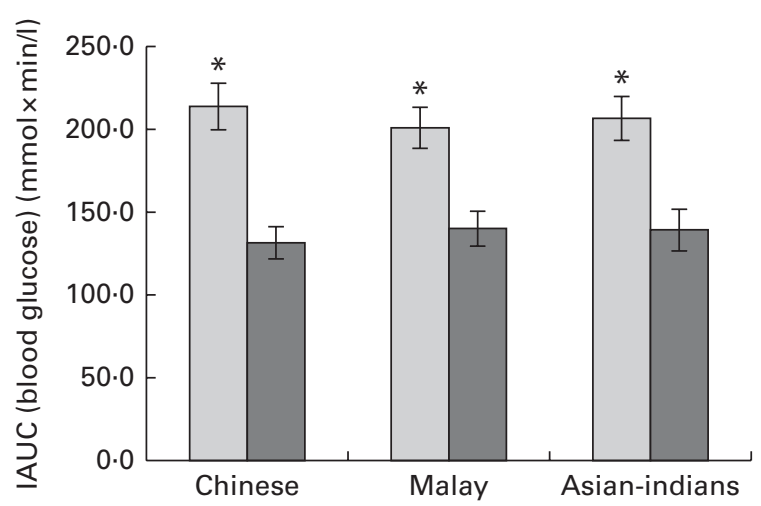

Fig. 5. Comparison of incremental AUC (IAUC) for glycaemic response between ethnic groups following consumption of Jasmine rice $(\square)$ and Basmati rice $(\square)$. Values are means, with their standard errors represented by vertical bars. *Mean value was significantly different in IAUC glycaemic response between rice varieties for each ethnic group $(P<0.05)$.

resistance $^{(29)}$ that may underlie their greater-than-normal tendency to develop diabetes and CVD compared with Chinese and Malays ${ }^{(22,30,31)}$. In the pathogenesis of T2DM, both $\beta$-cell dysfunction and decreased insulin sensitivity play key roles ${ }^{(32)}$. If insulin sensitivity decreases, insulin secretory response of pancreatic $\beta$ cells must increase to preserve normal glucose tolerance. This was evidenced by significantly higher HOMA-IR (a steady-state measure of insulin sensitivity) and HOMA-B (a measure of pancreatic $\beta$-cell function) among the Asian-Indians compared with Chinese and Malays. Chronic elevated insulin levels lead to impaired action and an exhaustion of pancreatic $\beta$ cells $^{(33)}$. Further deterioration of $\beta$-cell function and insulin sensitivity will result in worsening of glucose tolerance and risk for $\mathrm{T}_{2} \mathrm{DM}^{(33,34,35)}$. Although the GI values for each rice variety were similar between the ethnic groups, the same rice variety presented different stress to Asian-Indians and their pancreatic $\beta$ islet cells, resulting in higher compensatory IR for both Jasmine rice and Basmati rice, when compared with the Malays and Chinese. This may thus be an important contributing factor to the higher risk of T2DM in this ethnic group.

We did not find any significant differences in GI and II values to Jasmine rice and Basmati rice between Chinese, Malays and Asian-Indians. The present study lends support to the findings of previous research concerning the influence of ethnicity in GI values of foods. In the UK, there were no difference in GI values of biscuits and breakfast cereals between Asian-Indians and Caucasians ${ }^{(3)}$. Using the standard classification for low $(\leq 55)$, medium (56-69) and high GI $(\geq 70)^{(36-38)}$, the overall GI for Jasmine rice was classified as high GI, while Basmati rice was considered medium GI in the present study. While it is known that Jasmine rice, with its low amylose content $(11-18 \%)^{(39)}$, elicits a greater GR and a corresponding greater IR, it was unexpected that Basmati rice, with an intermediate amylose rice content $(20-25 \%)^{(40)}$ and claimed low GI on its packaging, was classified as medium GI in the present study (overall group mean as well as ethnicity mean). In Singapore, there is a lack of GI database for locally tested foods, and thus, there is a pressing need to uncover rice varieties that are truly low GI since rice in the major carbohydrate staple in this country ${ }^{(7)}$.

In the present study, we observed no differences in II values of both rice varieties despite ethnic differences in IR. The IR is a biological response to fluctuations in blood glucose levels. The II, however, is an index of the insulinaemic effects of food. The computation of II normalises an individual's IR to a test food against a reference food, similar to the computation of the GI. Therefore, while IR may differ, II, in contrast, remained consistent between individuals. The present study showed that both GI and II are properties of food, not influenced by the anthropometric measures and metabolic status of the participants. We showed that both GI and II were similar between ethnic groups regardless of their degree of insulin sensitivity and adiposity. Recent evidence has also shown that II values of rice were not different between healthy, hyperinsulinaemic and T2DM individuals, although the II was correlated with metabolic status ${ }^{(41)}$.

Our findings and existing evidence have shown that consumption of a high-GI food such as Jasmine rice leads to higher postprandial GR, more rapid decline in glucose levels from 60 min onwards and a correspondingly augmented IR compared with a lower-GI food ${ }^{(42)}$. A recent study has demonstrated that consumption of a high-GI meal led to a rapid decline in blood glucose levels ${ }^{(43,44)}$. In addition, brain activity is increased in regions related to food intake, reward and craving in the late postprandial phase ${ }^{(45)}$. This triggered excessive hunger and a preference for foods, such as high-GI foods that rapidly restored blood glucose to normal ${ }^{(46,47)}$. This could lead to overeating and contribute to problems such as overweight and obesity. These findings have considerable potential clinical significance given the global epidemic of obesity and T2DM and the especially high rates in Asian countries ${ }^{(1)}$, where white rice is the major contributor to the overall glycaemic load of the $\operatorname{diet}^{(7-10)}$. There was growing evidence that higher white rice consumption $(\geq 300-420 \mathrm{~g} / \mathrm{d})$ had been associated with a significantly increased risk of T2DM, especially in Asian populations ${ }^{(9,10,12)}$. Despite the positive findings of rice consumption and increased

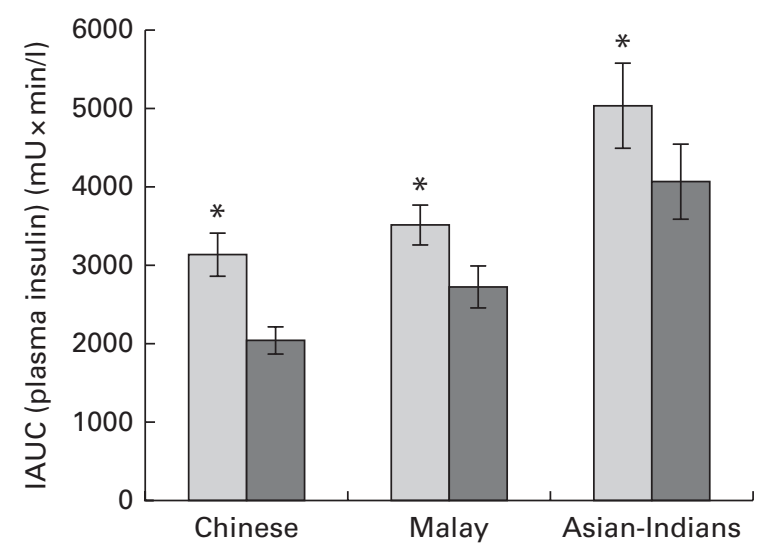

Fig. 6. Comparison of incremental AUC (IAUC) for plasma insulin response between ethnic groups following consumption of Jasmine rice ( $\square$ ) and Basmati rice $(\square)$. Values are means, with their standard errors represented by vertical bars. * Mean value was significantly different in IAUC insulin response between rice varieties for each ethnic group $(P<0.05)$. To convert insulin in $\mathrm{mU} / \mathrm{l}$ to $\mathrm{pmol} / \mathrm{l}$, multiply by 6.945 . 
risk of T2DM, rice intake was not associated with diabetes in a Chinese population ${ }^{(48)}$ and added no additional risk of CVD in Japanese men and women ${ }^{(49)}$. Therefore, evidence is still equivocal on rice intake and risk of T2DM in Asian populations.

Nonetheless, rice is a major dietary staple in Asian populations, and it appeared that Asian populations were more susceptible to the adverse effects of high intakes of white rice compared with the Western populations ${ }^{(10)}$. In Singapore, the mean rice intake among the population was 361.8 (SD 5.7$) \mathrm{g} / \mathrm{d}$, with Chinese, Malay and Asian-Indians consuming 364.9 (SD 6.5), 348.1 (SD 15.5) and 352.7 (SD 19.2) g, respectively ${ }^{(7)}$. This puts the population as being high consumers of white rice. It is, therefore, prudent to advice on consumption of rice with a lower GI that may reduce the glycaemic load of the diet. Additionally, a lower IR can help to improve long-term metabolic control in individuals who are insulin resistant and thereby reducing the future risk of T2DM.

The International Tables of the Glycaemic Index ${ }^{(50)}$ reported a wide range of GI values for different rice varieties, due to differences in the ratio of amylose to amylopectin, physical and chemical characteristics, other botanical structures and processing methods ${ }^{(51-55)}$. GI values vary even when considering the same variety of rice. For example, the GI values for Jasmine rice range from 48 to 109 and for Basmati rice from 43 to $69^{(50,56)}$. As the composition of rice and other carbohydrate-containing foods may vary according to where they are grown, the recommendation that the GI of such foods are best tested locally ${ }^{(36)}$. In the present study, we have used two locally available and commonly consumed rice varieties among the ethnic groups. The cooking method used was as per the manufacturers' instructions and represented how the population would cook these two rice varieties. There is currently no cut-offs nor classification for II values to food. Future studies can thus focus on establishing the corresponding II values when determining the GI of foods for greater clinical utility.

The strengths of the present study are that it measured the GR of the three ethnic groups fed identical foods within a common environmental milieu. We standardised the cooking method by using the exact amount of water to cook each rice variety in the same rice cooker to ensure consistency in rice texture. Using a standardised cooking method also allows a valid and unbiased comparison to be made between ethnic groups by removing the confounding effects of cooking methods. Moreover, we simultaneously measured the corresponding IR to gain a better understanding of the interplay between postprandial GR and IR as well as to determine both the GI and II values. However, we recognised that the limitation of the present study was that we have only tested two rice varieties and it may not be feasible to extrapolate these findings to all carbohydrate foods. However, these rice varieties were the most commonly consumed among the ethnic groups in Asia and are the major contributors to the overall glycaemic load of the Asian diet.

In summary, the present study has shown that there were no significant differences in the GI and II values to two commonly eaten rice varieties between the three ethnic groups. This suggests that GI values of Jasmine rice and Basmati rice determined in the present study are applicable to all the three ethnic groups in Singapore. Although there are currently no cut-offs for II values, the present results showed that II is a property of food, independent of the metabolic status of the individuals. Future studies testing the GI of foods may include testing the concurrent IR to derive the II for greater clinical utility in the prevention and management of T2DM. Despite similar GI values between the ethnic groups, the same rice presented greater pancreatic $\beta$-cell stress to Asian-Indians, resulting in higher compensatory IR, and possibly conferring a higher risk of T2DM in this ethnic group. Preserving $\beta$-cell function in at-risk populations is a critical factor in the prevention of T2DM onset ${ }^{(57)}$. Therefore, therapeutic prevention strategies should focus on encouraging the consumption of rice varieties with lower GI especially among the high-risk groups to maintain glucose homeostasis.

\section{Acknowledgements}

The authors extend their appreciation to Temasek Polytechnic interns Lee Yee Chin and Sarah Tan for assisting in the logistic aspects of the present study.

The present study was supported by the Singapore Institute for Clinical Sciences, A*STAR.

The authors' contributions are as follows: V. M. H. T., C. J. H. and Y. S. L. contributed to the conception and design of the study; V. M. H. T. and T. W. carried out the study; V. M. H. T. was responsible for the analysis of the blood samples, carried out the statistical analysis and drafted the manuscript. All authors critically reviewed the content of the manuscript and approved the final manuscript.

None of the authors has any conflict of interest to declare.

\section{References}

1. Chan JC, Malik V, Jia W, et al. (2009) Diabetes in Asia: epidemiology, risk factors, and pathophysiology. JAMA $\mathbf{3 0 1}$, 2129-2140.

2. Dickinson S, Colagiuri S, Faramus E, et al. (2002) Postprandial hyperglycemia and insulin sensitivity differ among lean young adults of different ethnicities. J Nutr 132, 2574-2579.

3. Henry CJ, Lightowler HJ, Newens K, et al. (2008) Glycaemic index of common foods tested in the UK and India. Br J Nutr 99, 840-845.

4. Venn BS, Williams SM \& Mann JI (2010) Comparison of postprandial glycaemia in Asians and Caucasians. Diabet Med 27, 1205-1208.

5. Kataoka M, Venn BJ, Williams SM, et al. (2013) Glycaemic responses to glucose and rice in people of Chinese and European ethnicity. Diabet Med 30, e101-e107.

6. Pawlak DB, Kushner JA \& Ludwig DS (2004) Effects of dietary glycaemic index on adiposity, glucose homoeostasis, and plasma lipids in animals. Lancet 364, 778-785.

7. Ministry of Health Singapore (MoHS) (2010) National Nutrition Survey 2010. http://www.moh.gov.sg/content/moh_ web/home/Publications/Reports/2011/national_health_ survey2010.html (accessed September 2014).

8. Murakami K, Sasaki S, Takahashi Y, et al. (2006) Dietary glycemic index and load in relation to metabolic risk factors in 
Japanese female farmers with traditional dietary habits. $A m J$ Clin Nutr 83, 1161-1169.

9. Villegas R, Liu S, Gao YT, et al. (2007) Prospective study of dietary carbohydrates, glycemic index, glycemic load, and incidence of type 2 diabetes mellitus in middle-aged Chinese women. Arch Intern Med 167, 2310-2316.

10. Hu EA, Pan A, Malik V, et al. (2012) White rice consumption and risk of type 2 diabetes: meta-analysis and systematic review. BMJ 344, e1454.

11. Yoon KH, Lee JH, Kim JW, et al. (2006) Epidemic obesity and type 2 diabetes in Asia. Lancet 368, 1681-1688.

12. Nanri A, Mizoue T, Noda M, et al. (2010) Rice intake and type 2 diabetes in Japanese men and women: the Japan Public Health Center-based Prospective Study. Am J Clin Nutr 92, 1468-1477.

13. Wolever TM, Jenkins DJ, Jenkins AL, et al. (1991) The glycemic index: methodology and clinical implications. Am J Clin Nutr 54, 846-854.

14. Jenkins DJ, Wolever TM, Taylor RH, et al. (1981) Glycemic index of foods: a physiological basis for carbohydrate exchange. Am J Clin Nutr 34, 362-366.

15. Chan HM, Brand-Miller JC, Holt SH, et al. (2001) The glycaemic index values of Vietnamese foods. Eur J Clin Nutr $\mathbf{5 5}$ 1076-1083.

16. Wolever TM, Jenkins AL, Vuksan V, et al. (2009) The glycaemic index values of foods containing fructose are affected by metabolic differences between subjects. Eur J Clin Nutr 63, 1106-1114.

17. Miller JB, Pang E \& Broomhead L (1995) The glycaemic index of foods containing sugars: comparison of foods with naturally-occurring $v$. added sugars. Br J Nutr $\mathbf{7 3}$, 613-623.

18. Holt SH, Miller JC \& Petocz P (1997) An insulin index of foods: the insulin demand generated by $1000-\mathrm{kJ}$ portions of common foods. Am J Clin Nutr 66, 1264-1276.

19. Reaven GM (2005) The insulin resistance syndrome: definition and dietary approaches to treatment. Annu Rev Nutr 25, 391-406.

20. Nilsson P, Nilsson JA, Hedblad B, et al. (2003) Hyperinsulinaemia as long-term predictor of death and ischaemic heart disease in nondiabetic men: The Malmo Preventive Project. J Intern Med 253, 136-145.

21. Takahashi F, Hasebe N, Kawashima E, et al. (2006) Hyperinsulinemia is an independent predictor for complex atherosclerotic lesion of thoracic aorta in non-diabetic patients. Atherosclerosis 187, 336-342.

22. Ministry of Health Singapore (MoHS) (2010) National Health Survey 2010. http://www.moh.gov.sg/content/dam/moh_ web/Publications/Reports/2011/NHS2010\%20-\%20low\% 20res.pdf (accessed June 2014).

23. Sun L, Ranawana DV, Leow MK, et al. (2014) Effect of chicken, fat and vegetable on glycaemia and insulinaemia to a white rice-based meal in healthy adults. Eur J Nutr $\mathbf{5 3}$, $1719-1726$

24. FAO/WHO (1998) Carbohydrates in Human Nutrition. Report of a Joint FAO/WHO Expert Consultation. Rome: Food and Agriculture Organization/World Health Organization.

25. Brouns F, Bjorck I, Frayn KN, et al. (2005) Glycaemic index methodology. Nutr Res Rev 18, 145-171.

26. Ranawana V, Clegg ME, Shafat A, et al. (2011) Postmastication digestion factors influence glycemic variability in humans. Nutr Res 31, 452-459.

27. Wolever TM, Brand-Miller JC, Abernethy J, et al. (2008) Measuring the glycemic index of foods: interlaboratory study. Am J Clin Nutr 87, 247S-257S.
28. Brand-Miller JC, Stockmann K, Atkinson F, et al. (2009) Glycemic index, postprandial glycemia, and the shape of the curve in healthy subjects: analysis of a database of more than 1000 foods. Am J Clin Nutr 89, 97-105.

29. Liew CF, Seah ES, Yeo KP, et al. (2003) Lean, nondiabetic Asian Indians have decreased insulin sensitivity and insulin clearance, and raised leptin compared to Caucasians and Chinese subjects. Int J Obes Relat Metab Disord 27, 784-789

30. Mak KH, Chia KS, Kark JD, et al. (2003) Ethnic differences in acute myocardial infarction in Singapore. Eur Heart J $\mathbf{2 4}$, $151-160$

31. Misra A \& Vikram NK (2004) Insulin resistance syndrome (metabolic syndrome) and obesity in Asian Indians: evidence and implications. Nutrition 20, 482-491.

32. Kahn SE (2003) The relative contributions of insulin resistance and beta-cell dysfunction to the pathophysiology of type 2 diabetes. Diabetologia 46, 3-19.

33. Hayashi T, Boyko EJ, Sato KK, et al. (2013) Patterns of insulin concentration during the OGTT predict the risk of type 2 diabetes in Japanese Americans. Diabetes Care 36, 1229-1235.

34. Lorenzo C, Wagenknecht LE, D'Agostino RB Jr, et al. (2010) Insulin resistance, beta-cell dysfunction, and conversion to type 2 diabetes in a multiethnic population: the Insulin Resistance Atherosclerosis Study. Diabetes Care 33, $67-72$.

35. Weyer C, Bogardus C, Mott DM, et al. (1999) The natural history of insulin secretory dysfunction and insulin resistance in the pathogenesis of type 2 diabetes mellitus. J Clin Invest 104, 787-794

36. Foster-Powell K, Holt SH \& Brand-Miller JC (2002) International table of glycemic index and glycemic load values: 2002. Am J Clin Nutr 76, 5-56.

37. Standards Australia (SA) (2007) Australian Standards - Glycemic Index of Foods, AS 4694-2007. Sydney: Standards Australia.

38. International Standards Organisation (ISO) (2010) Food products - determination of the glycaemic index (GI) and recommendation for food classification. ISO 26642:2010. https://www.iso.org/obp/ui/\#iso:std:iso:26642:ed-1:v1:en (accessed December 2014).

39. Suwansri S \& Meullenet JF (2004) Physicochemical characterization and consumer acceptance by Asian consumers of aromatic jasmine rice. J Food Sci 69, SNQ30-SNQ37.

40. Bhattacharjee P, Singhal RS \& Kulkarni PR (2002) Basmati rice: a review. Int J Food Sci Technol 37, 1-12.

41. Lan-Pidhainy X \& Wolever TM (2011) Are the glycemic and insulinemic index values of carbohydrate foods similar in healthy control, hyperinsulinemic and type 2 diabetic patients? Eur J Clin Nutr 65, 727-734.

42. Jenkins DJ, Kendall CW, Augustin LS, et al. (2002) Glycemic index: overview of implications in health and disease. $A m J$ Clin Nutr 76, 266S-273S.

43. Ludwig DS (2002) The glycemic index: physiological mechanisms relating to obesity, diabetes, and cardiovascular disease. JAMA 287, 2414-2423.

44. Ludwig DS, Majzoub JA, Al-Zahrani A, et al. (1999) High glycemic index foods, overeating, and obesity. Pediatrics 103, E26.

45. Lennerz BS, Alsop DC, Holsen LM, et al. (2013) Effects of dietary glycemic index on brain regions related to reward and craving in men. Am J Clin Nutr 98, 641-647. 
46. Campfield LA, Smith FJ, Rosenbaum M, et al. (1996) Human eating: evidence for a physiological basis using a modified paradigm. Neurosci Biobehav Rev 20, 133-137.

47. Strachan MW, Ewing FM, Frier BM, et al. (2004) Food cravings during acute hypoglycaemia in adults with type 1 diabetes. Physiol Behav 80, 675-682.

48. Yu R, Woo J, Chan R, et al. (2011) Relationship between dietary intake and the development of type 2 diabetes in a Chinese population: the Hong Kong Dietary Survey. Public Health Nutr 14, 1133-1141.

49. Eshak ES, Iso H, Yamagishi K, et al. (2014) Rice consumption is not associated with risk of cardiovascular disease morbidity or mortality in Japanese men and women: a large populationbased, prospective cohort study. Am J Clin Nutr 100, 199-207.

50. Atkinson FS, Foster-Powell K \& Brand-Miller JC (2008) International tables of glycemic index and glycemic load values: 2008. Diabetes Care 31, 2281-2283.

51. Panlasigui LN, Thompson LU, Juliano BO, et al. (1991) Rice varieties with similar amylose content differ in starch digestibility and glycemic response in humans. Am J Clin Nutr 54, 871-877.

52. Goddard MS, Young G \& Marcus R (1984) The effect of amylose content on insulin and glucose responses to ingested rice. Am J Clin Nutr 39, 388-392.

53. Larsen HN, Christensen C, Rasmussen OW, et al. (1996) Influence of parboiling and physico-chemical characteristics of rice on the glycaemic index in non-insulin-dependent diabetic subjects. Eur J Clin Nutr 50, 22-27.

54. Larsen HN, Rasmussen OW, Rasmussen PH, et al. (2000) Glycaemic index of parboiled rice depends on the severity of processing: study in type 2 diabetic subjects. Eur J Clin Nutr 54, 380-385.

55. Miller JB, Pang E \& Bramall L (1992) Rice: a high or low glycemic index food? Am J Clin Nutr 56, 1034-1036.

56. SUGIRS (2011) Glycemic index. http://www.glycemicindex. com (accessed July 2012).

57. Defronzo RA (2009) Banting lecture. From the triumvirate to the ominous octet: a new paradigm for the treatment of type 2 diabetes mellitus. Diabetes 58, 773-795. 